\title{
Demonstration of a fibre-based round trip correction system for the distribution of local oscillator signals to outstations of a radio telescope
}

\author{
R. McCool, M. Bentley, S.T. Garrington
}

\author{
Jodrell Bank Observatory, Macclesfield, Cheshire, SK11 9DL, UK.
}

\begin{abstract}
This paper provides details of the demonstration of a local oscillator distribution system using optical fibres as a transmission medium. The motivation for this work comes from a desire to demonstrate a fibre-based link for transferring local oscillator phase, for timing purposes, to outstations of the SKA (Square Kilometre Array).
\end{abstract}

\section{Introduction}

Any LO distribution system for the SKA needs to be capable of transmitting highly stable reference phase over long distances (100's of kilometres), reliably. The distances involved suggest that the signal will need repeating in-line over the transmission distance.

The use of an LO distribution system over fibre was successfully demonstrated in this study. Stabilities of; 1 ps in 1 second, 2 ps in 1 minute and 4 ps in 10 minutes were achieved. Installed fibre links were shown to be reciprocal in nature over the timescales of interest and therefore a suitable medium for the implementation of a round trip correction system of the type currently used in radio interferometers. Links of, up to, $110 \mathrm{~km}$ of fibre were demonstrated and the use of repeaters inline had no detrimental effect on the performance of the system. We therefore conclude that this type of system has possible applications in the distribution of LO and timing for the SKA.

\section{Alternative methods of time transfer}

There are a number of different ways in which time signals may be transferred. The current MERLIN system uses the microwave links very successfully with a stability of $1 \mathrm{ps}$ in one second. Other time transfer methods exist such as:

- Global Positioning System (GPS), Common View (CV) \& Carrier Phase (CP) methods,

- Two Way Satellite Time Transfer (TWSTT),

- Fibre LO distribution systems, such as those used in the EVLA or ALMA

Table 1 shows the typical, quoted, stability for these systems. The distribution of a hydrogen maser frequency standard over microwave or fibre links is the most stable method of LO distribution. Those LO distribution systems over fibre, in current usage, have been demonstrated, only, over short distances. Our objective was to demonstrate LO distribution over longer distances and repeatered hops. We used the MERLIN LO distribution system, using installed fibre links instead of microwave links as a test bed for our studies.

\section{Current MERLIN L-Band link system}

The MERLIN telescope is an array of seven radio telescopes spread across central and northern England. With a maximum separation of $217 \mathrm{~km}$, MERLIN provides an angular resolution of 50 millarcseconds at its prime operating frequency of $5 \mathrm{GHz}$ MERLIN, based at Jodrell Bank, achieves coherent operation using a frequency standard transmitted over microwave links. This system is locally known as the 'L-band Link' (LBL).

A Sigma-Tau hydrogen maser source provides the frequency standard for the LBL. Pulsed 1486.3 MHz signals are transmitted, 88 times per second, to each outstation. In order to avoid confusion between the LBL systems of different outstations, they are allocated different pulse lengths. Terminal equipment at the outstations contains excellent quality crystal oscillators that use the $1486.3 \mathrm{MHz}$ signal to lock a $5 \mathrm{MHz}$ frequency standard. Software records the difference in the go and return phase values and keeps a record of this information.

The use of a common frequency, the short timescales and the dispersion-less transmission medium, ensure that reciprocity applies and that changes in the returned phases are double the one-way changes. A correction, equal to, the difference on the go and return phase values, halved, is applied to data received from the different antennas. This corrects for the variable delay between antennas at distances of hundreds of kilometres from a central correlator. Tests in the laboratory and astronomical observations have confirmed that the LBL detects changes of delay at approximately the 1 picosecond level over periods of time extending to many hours.

\section{A fibre based LBL system}

The LBL system has been working reliably for over 20 years. However, a local oscillator distribution system using microwave links is not considered practical for the SKA, where the concentration of antenna would create unacceptable complexity and produce interference in the radio astronomy. Although transmissions in an optical fibre suffer dispersion the same TDM technique might offer the prospect of a comparable accuracy over fibre provided that similar-enough optical wavelengths are used in the two directions. 
Table 1: Alternative methods of local oscillator distribution

\begin{tabular}{|c|c|c|c|}
\hline System type & Distance & Stability & Ref \\
\hline MERLIN Microwave & $300 \mathrm{~km}$ & $1 \mathrm{ps}$ (in $1 \mathrm{~s}$ ) & \\
\hline GPS Common View & Intercontinental & $\begin{array}{l}3 \mathrm{~ns} \text { (in } 10^{3} \mathrm{~s} \text { ) - studies } \\
\text { not concerned with } \\
\text { stability in } 1 \mathrm{~s} .\end{array}$ & Weiss et al. (2005) \\
\hline GPS Carrier Phase & Intercontinental & $\begin{array}{l}0.7 \mathrm{ps}\left(\text { in } 10^{3} \mathrm{~s}\right)-\text { studies } \\
\text { not concerned with } \\
\text { stability in } 1 \mathrm{~s} .\end{array}$ & Plumb et al. (2005) \\
\hline TWSTT & Intercontinental & $\begin{array}{l}3 \text { ps (in } 1 \text { s) (best quoted } \\
\text { result, } 20-30 \text { ps more } \\
\text { commonly quoted) }\end{array}$ & Bardin et al. (2005) \\
\hline EVLA Fibre LO & $22 \mathrm{~km}$ fibre & $0.6 \mathrm{ps}$ (in $1 \mathrm{~s}$ ) & Durand et al. (2006) \\
\hline ALMA Fibre LO & $10 \mathrm{~km}$ fibre & $0.1 \mathrm{ps}$ (in $1 \mathrm{~s}$ ) & Kiuvhi et al. (2007) \\
\hline e-MERLIN specification & $\begin{array}{l}400 \mathrm{~km} \text {, using } 4 \text { repeater } \\
\text { sites, } 120 \mathrm{~km} \text { fibre } \\
\text { maximum single link } \\
\text { length. }\end{array}$ & $1 \mathrm{ps}$ (in $1 \mathrm{~s}$ ) & \\
\hline
\end{tabular}

The preliminary specifications for the reciprocity of a local oscillator system over fibre for the SKA are :

- 1 ps over a 1 second timescale (equivalent to 8 of phase at $22 \mathrm{GHz}$ - this is to keep signal loss at less than $1 \%$ over timescales less than a typical correlator integration period.

- 2 ps over timescales of 1 minute, to keep coherence loss to a few percent whilst integrating on a calibration source.

- 10 ps over a timescale of 10 minutes in order to maintain the linear phase variation between calibrator points.

The specifications are concerned with, not just the amplitude of changes in the system, but the speed of that change. Large changes can be corrected if they occur slowly over time. It is the amplitude of fast changes which are of particular concern, here.

The r.m.s stability of these changes was calculated by multiplying the Allan deviation by the sample time period of interest i.e.

$$
\sigma(\tau)=\tau \sigma_{y}
$$

The pre-existing, radio based LBL system was adapted to measure changes in the round trip delay in installed fibre of up to $110 \mathrm{~km}$ long the details of the experimental setup have been published previously McCool et al. (2008a). Various laser types were used in the experiments to investigate the effects on the stability of the system. These included lasers at 1310 $\mathrm{nm}$, the dispersion minimum of standard single mode fibre and lasers at $1550 \mathrm{~nm}$, the attenuation minimum of fibre with and without thermal control. In all cases, where the link was not attenuation limited, reciprocity applied and we achieved our stability goals. For the very longest links $1550 \mathrm{~nm}$ lasers with thermal control were found to be the most stable over the longest timescales McCool et al. (2008b). The results from measurements of the error in the round trip correction of the LBL, made with $1310 \mathrm{~nm}$ lasers on short links and $1550 \mathrm{~nm}$ lasers with thermal control on long links are shown in Table 2.

\section{Interferometry with a fibre LBL}

The MERLIN array was used to examine the effectiveness of a fibre LO distribution system and to demonstrate that such a system is suitable for interferometry at frequency ranges applicable to the SKA. An LO distributed over fibre was implemented on a baseline between Pickmere and Jodrell Bank. The distribution of antennae in the MERLIN array is shown in Fig. 1 and the baseline length between antennae can be deduced from the figure. The optical link was $28.6 \mathrm{~km}$ in length. The optical LO link was used for interferometry over a 12 month period and demonstrated the required stability over that time. The next step was to examine if a repeatered link would perform as well.

A repeatered link was set up for a fibre LO distribution system. This incorporated a $1550 \mathrm{~nm}$ optical signal from a thermally controlled laser, modulated with a frequency reference signal locked to a Hydrogen maser. The fibre links started at Jodrell Bank then connected to a loop of $28.6 \mathrm{~km}$ of installed fibre, an in-line repeater at Jodrell Bank received, then retransmitted the signal over a further $35 \mathrm{~km}$ of installed fibre before it was received by a unit at the Darnhall antenna. A direct com- 
Table 2: Stability of an LO over fibre link when compared to a system with no transmission.

\begin{tabular}{llll} 
Timescale & $\begin{array}{l}\text { Back to Back stability } \\
\text { measured using only } \\
\text { terminal equipment, no } \\
\text { optics }\end{array}$ & $\begin{array}{l}\text { Stability of a } 1310 \mathrm{~nm} \\
\text { laser, no thermal control } \\
\text { over } 28.6 \mathrm{~km} \text { of fibre }\end{array}$ & $\begin{array}{l}\text { Stability of a } 1550 \mathrm{~nm} \\
\text { laser with thermal } \\
\text { control over } 110 \mathrm{~km} \text { of } \\
\text { fibre }\end{array}$ \\
\hline $1 \mathrm{~s}$ & $\begin{array}{l}1 \mathrm{ps} \mathrm{r.m.s} \\
1 \mathrm{ps} \text { rams }\end{array}$ & $\begin{array}{l}1 \mathrm{ps} \mathrm{r.m.s} \\
2 \mathrm{ps} \text { r.m.s }\end{array}$ & $\begin{array}{l}1 \mathrm{ps} \text { r.m.s.s } \\
2 \mathrm{ps} \text { r.m.s }\end{array}$ \\
\hline
\end{tabular}

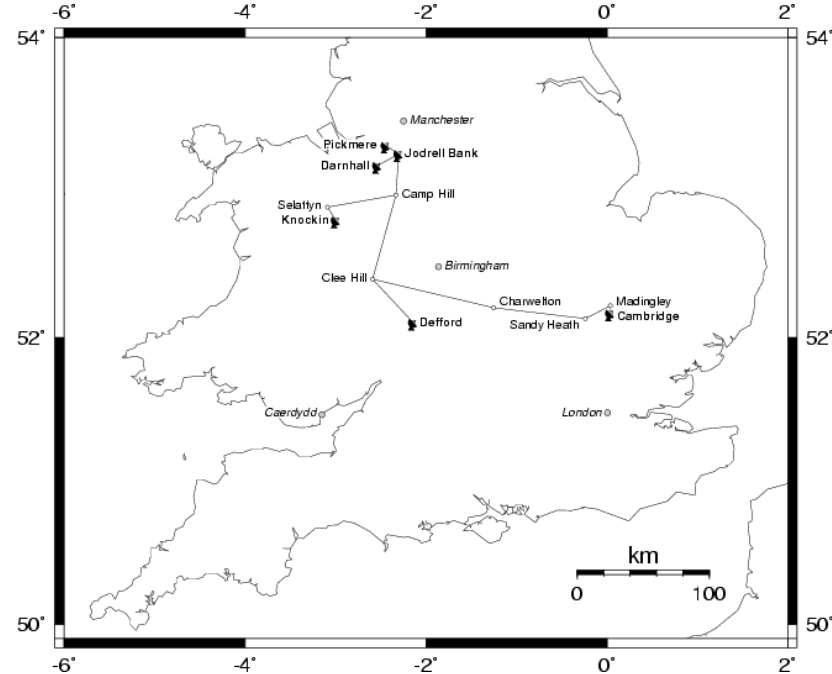

Fig. 1: Distribution of antennae in the MERLIN array.

parison between the existing fibre $\mathrm{LO}$ and the microwave $\mathrm{LO}$ is not possible, because the antenna can only accept one LO at a time. Therefore alternative schemes were used to compare the two systems.

The first comparison involved the measurement of astronomical phases for observations on the same baseline, but at different times, firstly using a microwave LO and then using a repeatered fibre LO. This allowed a direct comparison on the two different methods of LO distribution using the same antenna. The baseline used was the Defford-Pickmere baseline. The measured phases extracted from MERLIN are shown in Fig. 2. The corresponding Allan deviation plot is shown in Fig. 3.

The stability of the astronomical phase is dominated by tropospheric and ionospheric effects. In order to attempt a comparison under the same atmospheric conditions, the second comparison measured astronomical phases for same observation using two different baselines. These baselines were similar in separation but one served by a microwave LO and the other with a repeatered fibre LO. The baselines used were the Defford-Pickmere baseline and the Defford-Darnhall baseline. The measured phases extracted from MERLIN are shown in Fig. 4. The corresponding Allan deviation plot is shown in Fig. 5.

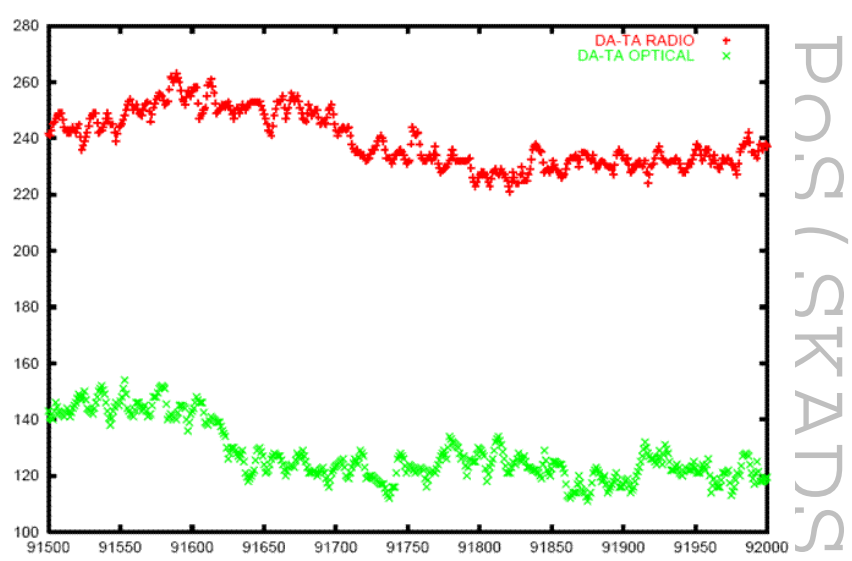

Fig. 2: Astronomical phase on the Defford-Pickmere baseline for a fibre LO (in green) and a microwave LO (in red)

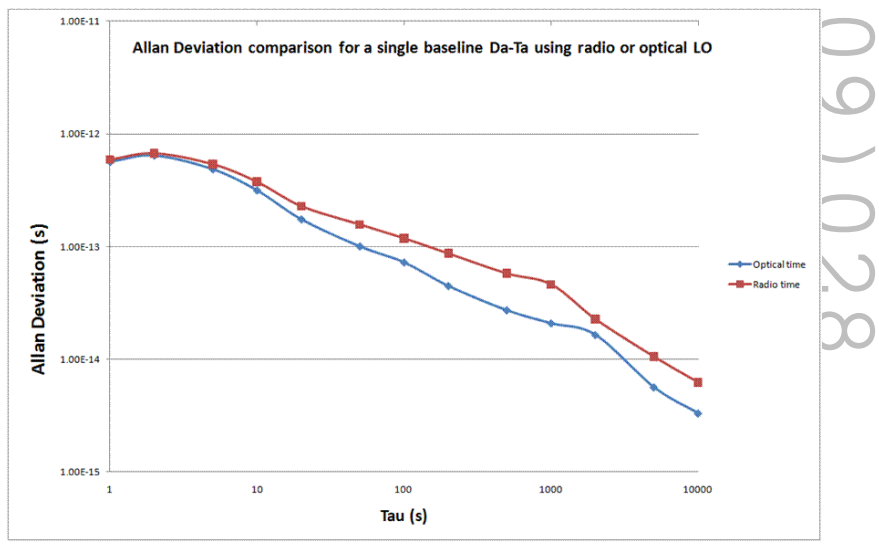

Fig. 3: Allan deviation plots for a fibre LO (in blue) and a microwave LO (in red) 


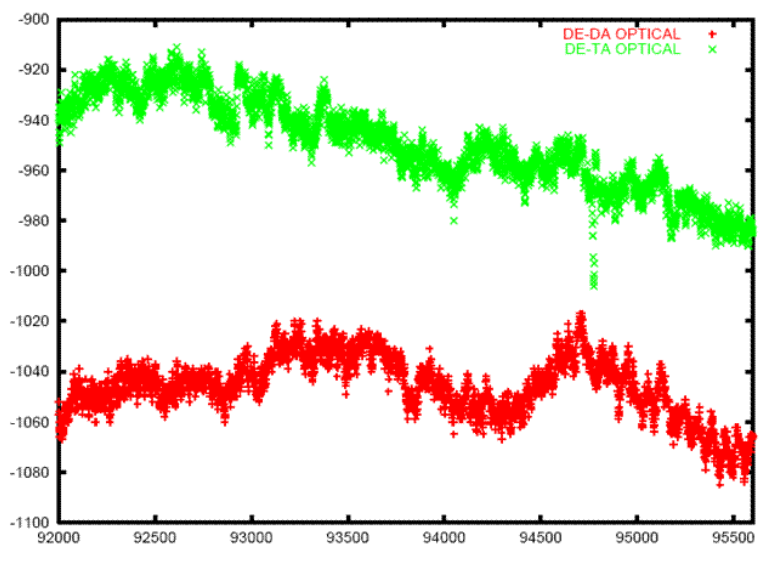

Fig. 4: Astronomical phase for the same observation Defford-Darnhall baseline with a microwave LO (in red) and Defford-Pickmere baseline for a fibre LO (in green).

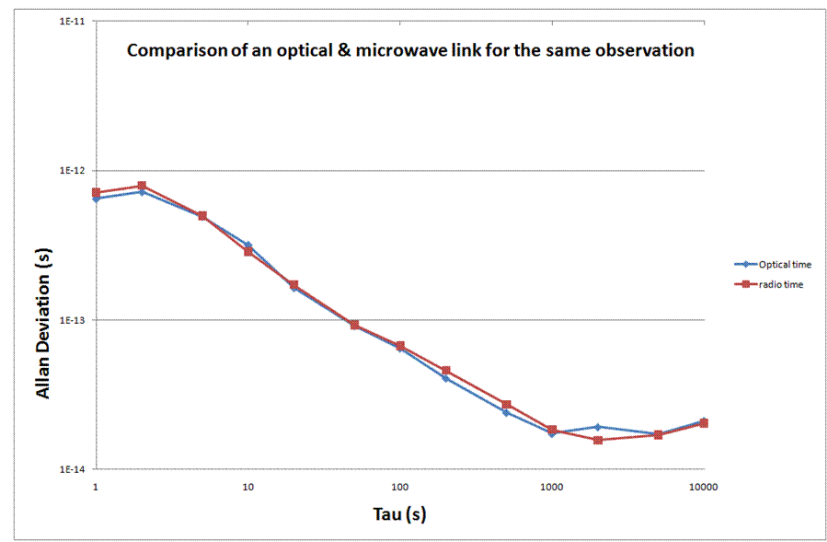

Fig. 5: Allan deviation plots for a fibre LO (in blue) and a microwave LO (in red)

\section{Conclusions}

The use of an LO distribution system over fibre was successfully demonstrated in this study. Stabilities of; 1 ps in 1 second, 2 ps in 1 minute and 4 ps in 10 minutes were achieved. Installed fibre links were shown to be reciprocal in nature over the timescales of interest and therefore a suitable medium for the implementation of a round trip correction system of the type currently used in radio interferometers. Links of, up to, $110 \mathrm{~km}$ of fibre were demonstrated and the use of repeaters inline had no detrimental effect on the performance of the system. We therefore conclude that this type of system has possible applications in the distribution of LO and timing for the SKA.

Acknowledgements. This effort is supported by the European Community Framework Programme 6, Square Kilometre Array Design Studies (SKADS), contract no 011938.

\section{References}

Bardin J., Weinreb S., Bagri D., An LO Phase Link Using a Commercial Geo-stationary Satellite, Jet Propulsion Laboratory, Jan 2005, http://trs-new.jpl .nasa.gov/ dspace/handle/2014/39600

Durand S., Jackson J., Morris K., Phase Coherence of the EVLA radio telescope, EVLA Memo 105, 2006 http://www.aoc.nrao.edu/evla/geninfo/ memoseries/evlamemo105.pdf

Kiuchi H., Iguchi S., Hasegawa T., Alternative Scheme Line Length corrector Prototype Test report Subsystem Laboratory test, ALMAJ-BE-LO-07003-A-REP, Draft Version, January 2007.

McCool R., Bentley M., Anderson B., Argo M., Spencer R. and Garrington S. T., "A report describing the transfer of a 1486.3 MHz frequency standard over $110 \mathrm{~km}$ of installed fibre," European Conference on Optical Communications Sept 2008.

McCool R., Bentley M., Garrington S.T, Spencer R., Davis R., Anderson B., "Phase transfer for radio astronomy interferometers, over installed fibre networks, using a round trip correction system," Precise Time and Time Interval Systems and Applications Meeting, Nov 2008.

Plumb J.F., Larson K.M, Long-Term Comparisons Between Two-Way Satellite and Geodetic Time Transfer Systems, IEEE transactions on ultrasonics, ferroelectrics, and frequency control, vol. 52, no. 11, November 2005.

Weiss M.A, Petit G., Jiang Z., "A Comparison of GPS Common-View Time Transfer to All-in-View.," Proceedings of the 2005 IEEE International Frequency Control Symposium and Exposition, 2005. 